\title{
Clutter Simulation Overview
}

\author{
Yawei Liu ${ }^{1, \text { a) }}$, Zhiqiang $\mathrm{Li}^{2}$ \\ ${ }^{1}$ School of Ordnance Engineering College, Shijiazhuang 050003, China. \\ ${ }^{2}$ School of Ordnance Engineering College, Shijiazhuang 050003, China. \\ a) maojindao1228@sina.com
}

\begin{abstract}
Most of the current clutter simulations are based on theoretical models, radar emission wave and undulating ground, vegetation cover and other factors considered less, this could lead to lower degree of simulation. This paper presents a clutter simulation method based on digital terrain model, combined with ground data and radar emission waves to generate a more realistic ground clutter data.
\end{abstract}

Keywords: Clutter, Simulation, Model

\section{INTRODUCTION}

Radar target environment simulation is a combination of modern analog technology and radar technology. It is the establishment of radar system model, the use of hardware and software means to reproduce the radar system dynamic work process means. The radar simulator based on this technology is a powerful tool for debugging and testing radar and also can be widely used in radar military simulation training. Clutter simulation is an important part of the radar target environment simulation, and it is also an important factor in the evaluation of the environmental model in the system.

The existing clutter simulation model, most of them need to set the model parameters, and then produce a specific model of the clutter, such as Rayleigh model, logarithmic normal model, these models cannot effectively combine radar-transmitted wave and antenna-related features, such as radar sidelobe effects, etc., and cannot be based on real-time changes in terrain and geomorphology to re-change the model parameters, access to real-time closer to the real clutter model. Based on the above conditions and the status quo, this paper presents a simulation model of generated clutter based on digital terrain model, make it closer to the real natural environment to get more realistic simulation results.

\section{RESEARCH STATUS}

At present, the clutter is generally regarded as a random process, different clutter amplitudes conform to a specific probability distribution model. One of the main statistical properties of the clutter is clutter amplitude distribution characteristics, commonly used classical statistical models include Rayleigh distribution, Weibull distribution, and logarithmic normal distribution and so on.

\subsection{Rayleigh distribution model}

The Rayleigh distribution is suitable for describing meteorological clutter, chaff interference, ground clutter of low resolution radar (Antenna beam width greater than $2^{\circ}$, pulse width greater than 1 us ) and when the radar clutter in a clutter cell contains a large number of independent, no strong scattering point, the clutter envelope obeys 
the Rayleigh distribution. According to stochastic process theory, the orthogonal two-way signal of Rayleigh distribution clutter can be composed of two related Gaussian sequences.

If $x$ represents the envelope amplitude of the Rayleigh distribution clutter echo, the probability density function of $x$ is:

$$
\mathrm{p}(x)=\frac{x}{\delta^{2}} e^{-\frac{x^{2}}{2 \delta^{2}}}, \quad x \geq 0
$$

The distribution function is:

$$
\mathrm{F}(x)=1-e^{\frac{x^{2}}{2 \delta^{2}}}
$$

Where $\delta$ is the standard deviation of the clutter.

Since the noise in the radar target environment also follows the Rayleigh distribution, therefore this model can also be used to represent noise in a radar target environment.

The simulation results shown in Figure 1:
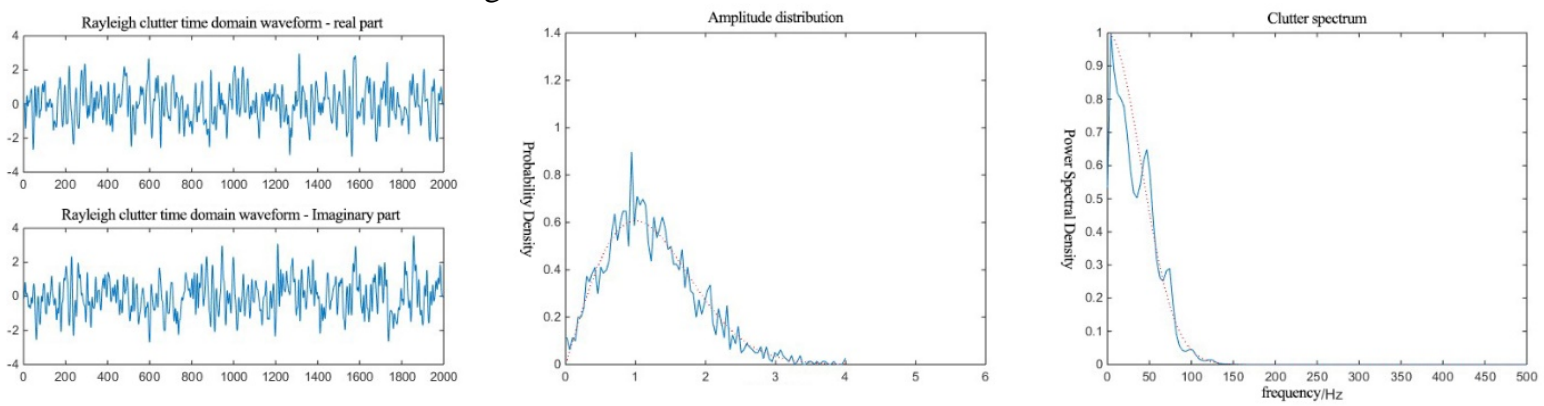

FIGURE 1. Rayleigh distribution clutter simulation.

\subsection{Logarithmic normal distribution model}

In many practical applications, when the high-resolution radar on the ground for low incidence angle detection, the distribution of clutter is obviously different from Rayleigh distribution, the probability density function of its clutter envelope will have a long tail, and with the ground ups and downs increased, the deviation between the actual clutter distribution and the Rayleigh distribution also increases. Through the analysis of the experimental results, when selecting the appropriate parameters, Log-Normal distribution and the measured curve can be a good fit, that is to say, with the improvement of radar discrimination ability, the tail of the clutter becomes longer and the backscattering characteristic deviates from the Rayleigh distribution and conforms to the Log-Normal distribution.

If $x$ represents the envelope amplitude of the Log-Normal distributed clutter echo, the probability density function of $X$ is:

The distribution function is:

$$
\mathrm{p}(x)=\frac{1}{\sqrt{2 \pi} \delta x} e^{-\frac{\left(\ln \frac{x}{\mu}\right)^{2}}{2 \delta^{2}}},(x>0, \delta>0, \mu>0)
$$

$$
\mathrm{F}(x)=\frac{1}{2}\left[1+\operatorname{erfc}\left(\frac{\ln \left(\frac{x}{\mu}\right)}{2 \sqrt{2} \delta}\right)\right]
$$

Where $\mu$ is the scale parameter, representing the mean of the distribution, $\delta$ is the shape parameter, indicating the deviation of the distribution, $\operatorname{erfc}(\ldots)$ is the residual error function. 
The trailing of the Log-Normal distribution increases with increasing $\delta$, the ratio of the mean to the median $\rho=e^{\frac{\delta^{2}}{2}}$ can be used to control the shape of the distribution, survey about the actual noise data show that: The value range of $\rho$ is generally $[1.065,1.93], \delta$ corresponds to a range of $[0.355,1.147]$.

Log-Normal distribution has a longer tail, and therefore apply to low incident angle, complex terrain clutter data or flat area high resolution ground clutter data.

The simulation results shown in Figure 2:
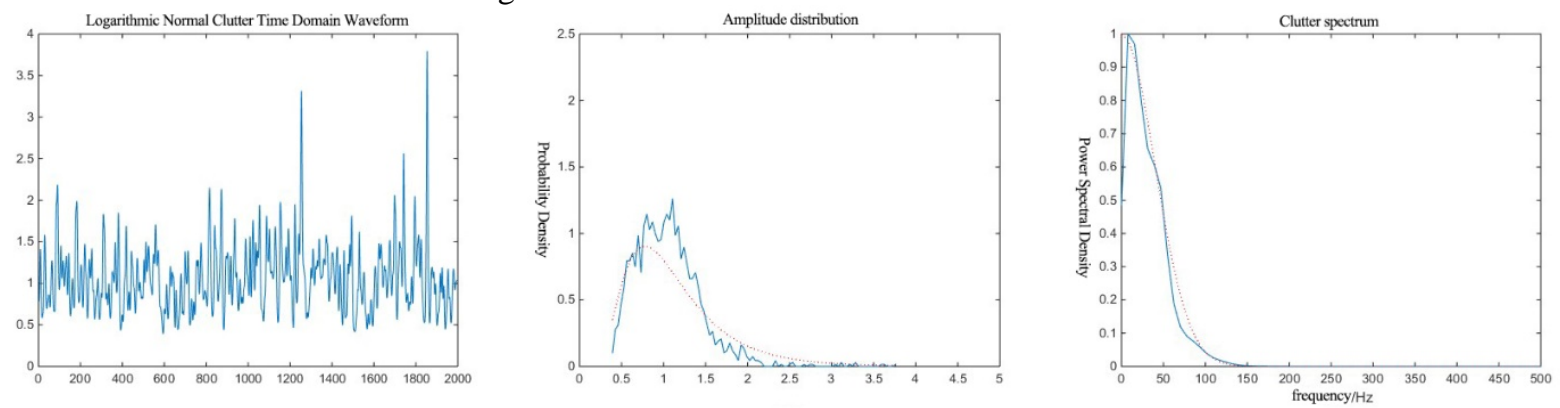

FIGURE 2. Logarithmic normal clutter simulation.

\subsection{Weibull distribution model}

Weibull distribution is the general case of Rayleigh distribution and Log-Normal distribution, the dynamic range is between the Log-Normal distribution and the Rayleigh distribution, so that the actual clutter distribution can be accurately represented in a wider range.

If $x$ is the envelope amplitude of the Weibull distribution clutter echo, the probability density function of $x$ is:

$$
\mathrm{p}(x)=\left(\frac{p}{q}\right)\left(\frac{x}{q}\right)^{p-1} e^{-\left(\frac{x}{q}\right)^{p}}, x \geq 0
$$

The distribution function is:

$$
\mathrm{F}(x)=1-e^{-\left(\frac{x}{q}\right)^{p}}
$$

Where $\mathrm{q}$ is the scale parameter, representing the mean of the distribution, $\mathrm{p}$ is the shape parameter, indicating the deviation of the distribution. When the $\mathrm{q}$ is fixed, the trailing of the Weibull distribution increases with the decrease of p. The Log-Normal distribution and the Rayleigh distribution are special examples of the shape parameter p of the Weibull distribution.

In general, terrestrial clutter can be described by Weibull distribution in the case of high resolution radar and low incidence angles.

The simulation results shown in Figure 3:
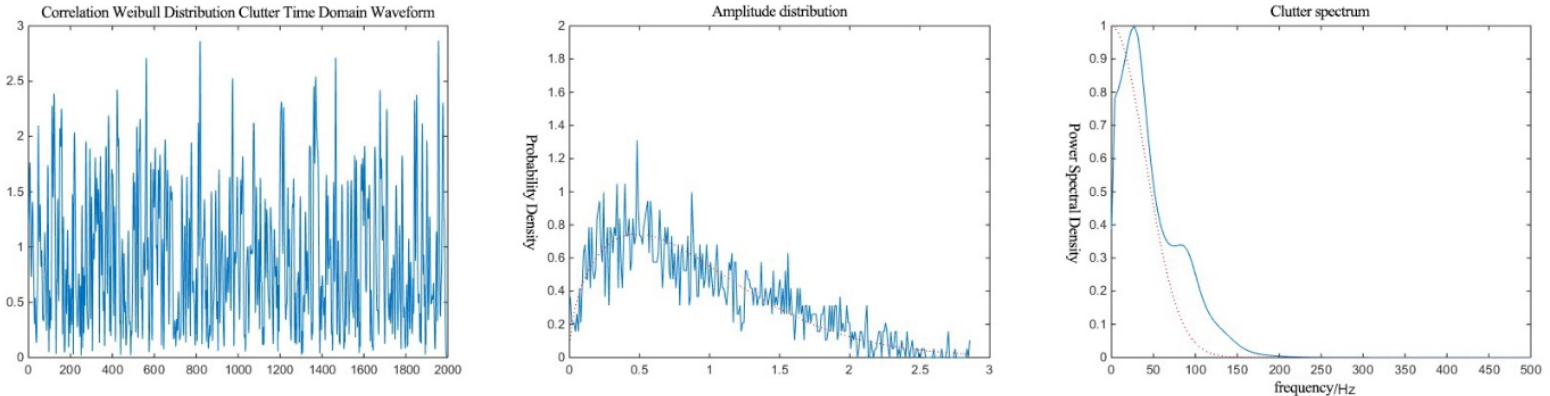

FIGURE 3. Weibull distribution clutter simulation. 


\subsection{Shortages}

(1) Directly using the classic model for simulation, without considering the terrain changes, so that the simulation results tend to idealized.

(2) There is no connection with the radar launch wave, ground clutter generated out of thin air, the result is not realistic.

\section{BASIC THEORY}

Set the radar to send the wave signal to $s(t)$, after a delay of $t_{d k}$, the clutter echo signal $c_{k}(t)$ of the Kth clutter tile is obtained. The following expression can be assumed:

$$
c_{k}(t)=L \cdot s\left(t-t_{d k}\right)
$$

Among them, $L$ is the voltage amplitude attenuation coefficient, including distance attenuation, gain of the antenna in this direction, RCS variation of clutter tiles, and the like.

The relationship between the transmit power and the received power:

$$
P_{r}=\frac{P_{t} G_{t} G_{r} \lambda^{2} \sigma}{(4 \pi)^{3} R^{4}}
$$

Whereby the attenuation coefficient $L$ is obtained:

$$
L^{2}=\frac{P_{r}}{P_{t}}=\frac{G_{t} G_{r} \lambda^{2} \sigma}{(4 \pi)^{3} R^{4}}
$$

Where the antenna gain $G$ varies with the angle, that is, the antenna pattern, you can get the relevant data through the table, $\sigma$ is the equivalent RCS of the clutter tile unit. Therefore, the final formula is:

$$
L^{2}=\frac{G_{t}(\theta, \phi) G_{r}(\theta, \phi) \lambda^{2} \sigma}{(4 \pi)^{3} R^{4}}
$$

RCS is equal to the product of the backscatter coefficient $\sigma_{0}$ and the ground clutter tile area, $\sigma_{0}$ is an important concept in the study of ground clutter properties, and is also the most basic research object, clutter characteristics of a key indicator. Many scholars at home and abroad through a large number of field experiments and experimental data obtained by the statistical analysis of a variety of empirical models, such as Morchin models.

Morchin proposed a posterior scattering empirical model for a variety of landforms, this model takes into account the reflection components of the vertical mirror, as follows:

$$
\sigma^{0}=\frac{A \sigma_{c}^{0} \sin \theta}{\lambda}+u \cot ^{2} \beta_{0} \exp \left[-\frac{\tan ^{2}(B-\theta)}{\tan ^{2} \beta_{0}}\right]
$$

Among them, $u=\sqrt{f_{0}} / 4.7, \theta_{c}=\arcsin \left(\lambda / 4 \pi h_{e}\right), h_{e}=9.3 \beta_{0}^{2.2}$. This model is suitable for a variety of landforms, such as desert, farmland, hills, mountains, etc., have a higher accuracy.

In addition there are $\gamma$ models, $\gamma-f$ models and so on. Choose the appropriate model will be able to get more accurate $\sigma_{0}$ value

By the above formula, ground clutter and radar launch wave, antenna pattern, equivalent RCS and other factors established contact, by doing so, and the more realistic clutter simulation results are obtained.

\section{CONCLUSION}

In summary, traditional research mainly uses statistical methods to extract clutter statistical characteristics, different clutter models are proposed for different irradiation environments and radar systems, such as Rayleigh distribution, lognormal distribution, Weibull distribution and so on. These models are obtained from the data itself 
and the statistical assumptions, they cannot effectively combine the radar emission and antenna pattern of the relevant characteristics, such as radar sidelobes, etc., and cannot be based on real-time terrain changes to change the model parameters, get real-time closer to the real clutter model, therefore, this paper presents a generation of clutter simulation model based on digital terrain model, and makes it closer to the real natural environment.

\section{REFERENCES}

1. David K. Barton. Radar Equations for Modern Radar [M].

2. BASSEM R. MAHAFZA. Radar Systems Analysis and Design using MATLAB 3rd Edition 2013[M].

3. LI Qing-hua, DENG Cheng, YAO Yun-ping. The Comparison of Correlated Non-Gaussian Radar Clutter Simulation Based on ZMNL and SIRP Method [J]. Electronic Information Countermeasure Technology, 2001,07

4. WANG Bo, LI Wan-yu. Simulation of Correlated Weibull Clutters Based on ZMNL [J]. Fire Control Radar Technology, 2008,03

5. DU Yong, LI Yi-lin, YANG Hai-ju. Modeling and Simulation of ZMNL-based Correlated Radar Clutter [J]. Fire Control Radar Technology, 2012,12

6. Yang Feng-feng, Zhou Zhi-min. Radar Clutter Simulation Based on ZMNL [J]. Modern Radar, 2003, 09.

7. ZHANG Peng-fei. Clutter Simulation of Airborne Radar System Based on CUDA [D]. Master's Thesis of Xidian University,2013

8. LU Jian-qi, Research on Modeling and Simulation for Rader Echo [D]. Master of Science in Information Engineering, 2006

9. JIANG Bin, HE Xiang, WANG Hong-qiang, LI Xiang. An ameliorated simulation method for the LogNormal clutter [J]. Electro-Optic and Control, 2006, 06.

10. Lü Yong. Analysis of Antenna Pattern Parameters [J]. Radar Technology, 2015.

11. CHEN Yuan, WU Wen, WANG Xiao-jun, CHEN Hui-yun. Simulation of Ground Clutter for Land-based Rader [J]. Ship Electronic Warfare, 2011, 02.

12. LI Xiang-ping, ZHANG Li-qiang, ZHAO Zhen-bo, YING Tao. Research on the Experience Model of Ground Clutter Backscatter Coefficient [J]. Ship Electronic Engineering, 2011.

13. Hong Li-na. Research on Rader Land Clutter Modeling and Simulation [D]. National Defense Science and Technology University master's degree thesis, 2003

14. ZHANG Jie, HE Qiang, HAN Zhuang-zhi. Generation of signal transmitted by a radar based on System Vue [J]. Modern Electronics Technology, 2014, 11

15. WEI Dan-ting, TASHPOLAT.Tiyip, LEI lei, ZHANG Fei, HAN Gui-hong, MAMATSAWUT, Simulation of Rader Backscattering Coefficient Image at Different Incidence Angle [J]. Remote sensing information, 2012, 06.

16. LI Xing. The Study on Terrain Scattering Characteristics and Paremeter Inversion and the Establishment of Statistical Model [J]; Xi'an University of Electronic Science and Technology, 2014.

17. CHEN Yan-hui, XIE Wei-xin.An stochastic fractal model of ground-sea clutter [J]. Journal of Electronics University of Science and Technology, 2000, 06.

18. FAN Guo-zhong, HUANG Zhi-qiang, ZHANG Xian-yi, YANG Zheng-long, Modeling and Simulation of ZMNL-based Correlated Radar Clutter [J]. Modern radar, 2010, 09. 